\title{
Measurement of ionization, attachment, detachment and charge transfer rate coefficients in dry air around the critical electric field
}

\author{
Journal Article \\ Author(s): \\ Hösl, Andreas (D); Häfliger, Pascal; Franck, Christian (1) \\ Publication date: \\ 2017-12-06 \\ Permanent link: \\ https://doi.org/10.3929/ethz-b-000219825
}

Rights / license:

In Copyright - Non-Commercial Use Permitted

Originally published in:

Journal of Physics D: Applied Physics 50(48), https://doi.org/10.1088/1361-6463/aa8faa 
This is an author-created, un-copyedited version of an article published in Journal of Physics D: Applied Physics. IOP Publishing Ltd is not responsible for any errors or omissions in this version of the manuscript or any version derived from it. The Version of Record is available online at https://doi.org/10.1088/1361-6463/aa8faa. 


\title{
Measurement of ionization, attachment, detachment and charge transfer rate coefficients in dry air around the critical electric field
}

\author{
A Hösl, P Häfliger and C Franck \\ E-mail: cfranck@ethz.ch \\ High Voltage Laboratory, Swiss Federal Institute of Technology, Swizerland
}

\begin{abstract}
We obtain pressure-dependent rate coefficients in dry synthetic air $\left(79 \% N_{2}, 21 \% \mathrm{O}_{2}\right)$ by fitting Pulsed Townsend measurements over a pressure range from 10 to $100 \mathrm{kPa}$, around the critical density-reduced electric field from 86 to $104 \mathrm{Td}$. The physical processes are reviewed and set in relation to a suitable kinetic model. A procedure for fitting kinetic reaction rates is described, which is based on finite-volume simulations of charge carrier drift. Electron attachment, ionization and detachment as well as ion conversion rate coefficients are obtained. We find a quadratic pressure dependency in the conversion rate, consistent with three-body collisions, and observe a pressure-dependency in the onset of electron avalanche growth in dry air.
\end{abstract}

dry air, swarm parameters, electric strength, Pulsed Townsend Experiment, gaseous insulation, rate coefficients, ion charge transfer

Submitted to: J. Phys. D: Appl. Phys.

\section{Introduction}

Air is widely used as an electrical insulation medium, and has recently attracted renewed interest as a climate-neutral alternative to $\mathrm{SF}_{6}$ in high-voltage gas-insulated switchgear (GIS) [1, 2]. Although the electric strength is lower compared to $\mathrm{SF}_{6}$, using air offers several advantages: it has no global warming potential and is cheaper. Moreover, toxicity of the gas after severe arcing is negligible compared to fluorinated gases.

Modeling and prediction of the breakdown behaviour requires a detailed understanding of the relevant physical processes at application pressure. The most relevant kinetic processes have been identified, but there is a significant spread in the obtained rates of different authors. Main contributions have been published by Frommhold [3, 4], Harrison and Geballe [5], Prasad [6], Moruzzi et al. [7], Verhaart and van der Laan [8, 9], Wen and Wetzer [10, 11], Friedrich[12], Kossy et al. [13] and Aleksandrow [14] between 1960 and 1995. A more recent theoretical paper has been published by Pancheshnyi in 2013 [15], in which the author calculates a pressure dependency in the density-reduced critical electric field strength $\left(E / N_{\text {crit }}\right.$ with electric field $E$ and particle density $N$ ) of synthetic air. 
Compared to previous, often decades old measurements, we benefit from major advancements in pulsed lasers, signal amplification, and voltage source technology as well as gas purity and computational resources. We are able to improve the accuracy of the respective rate coefficients, and qualitatively confirm the calculation of Pancheshnyi. We further present a rate-fitting method for pulsed Townsend experiments, based on simulation on graphics cards. A comparable approach has been published by Urquijo et al. [16], and Bekstein et al. [17], on CPUs.

The publication is structured as follows: The experimental setup is presented in section 2. The kinetic processes are reviewed in section 3, and compared to a model proposed by Verhaart and van der Laan [8,9], which is used in this publication. The results are presented in section 4 , where we compare our findings to the literature, both experimental data and Bolsig + calculations, which are based on tabulated cross-section data. Appendix A and B deal with the method and structure of the numerical simulation, where we describe the implementation of the simulation and fitting method, complemented by a convergence analysis.

\section{Experimental Setup}

In our Pulsed Townsend experiment the gas is subjected to a homogeneous electric field in between Rogowski-shaped electrodes. Electrons are released from a backilluminated photo-cathode by a pulsed UV laser of $266 \mathrm{~nm}$ wavelength at a repetition frequency of $20 \mathrm{~Hz}$. Those electrons travel a distance of $1-2 \mathrm{~cm}$ towards the anode, and interact with gas molecules. The displacement current is measured and evaluated for different electric field strength, pressure and electrode distance. Details about the setup can be found in [18], and we limit this section to specifics of the presented air measurements.

Exceeding our usual measurement pressure range, normally below $10 \mathrm{kPa}$, proved to be difficult. We found that deformation of the steel vessel walls due to pressure differences have to be taken into account, since it distorts the electrode spacing. Corrected via electron velocity measurements in pure nitrogen, as described in [19], we estimate the distance accuracy to be better than $100 \mu \mathrm{m}$ over the whole pressure range. The metal film on the photo-cathode that we used in previous work, palladium, exhibits a sharp decrease in quantum efficiency in the presence of oxygen. This effect is stronger at elevated oxygen pressure, which limits the lifetime of photo-cathodes drastically. Similar findings have been reported in [4]. Experimenting with two layer films, we found $\mathrm{Cu}-\mathrm{Pd}$ photo-cathodes with approximate thicknesses of 5 and $12 \mathrm{~nm}$ to show acceptable efficiency (roughly $10^{6}$ electrons per shot) and life-time.

Our amplifier-setup has a bandwidth $(3 \mathrm{~dB})$ of $175 \mathrm{MHz}$, and records with $10 \mathrm{GS} / \mathrm{s}$. This bandwidth is taken into account in the simulation: a digital low-pass filter was adapted in accordance with the specifications of the vendor.

The oxygen and nitrogen gas bottles have a purity of 5.0 and 6.0, respectively. The base pressure of the chamber is lower than $5 \cdot 10^{-6} \mathrm{~Pa}$. With opened gas inlet pipes, it is ensured that the pressure is below $0.05 \mathrm{~Pa}$ before filling the gas.

Figure 1 shows a measurement current at $100 \mathrm{kPa}$ on two different time-scales. A laser pulse releases electrons from the photo-cathode, which then travel to the anode on a time-scale in the order of $100 \mathrm{~ns}$. Slower ions, produced in ionization or attachment events, take up to $30 \mu \mathrm{s}$. The plot includes, as a result of the fitting procedure, the individual currents of the different species (discussed at the end of section 3), as well as their superposition, which is the displacement-current we measure. 

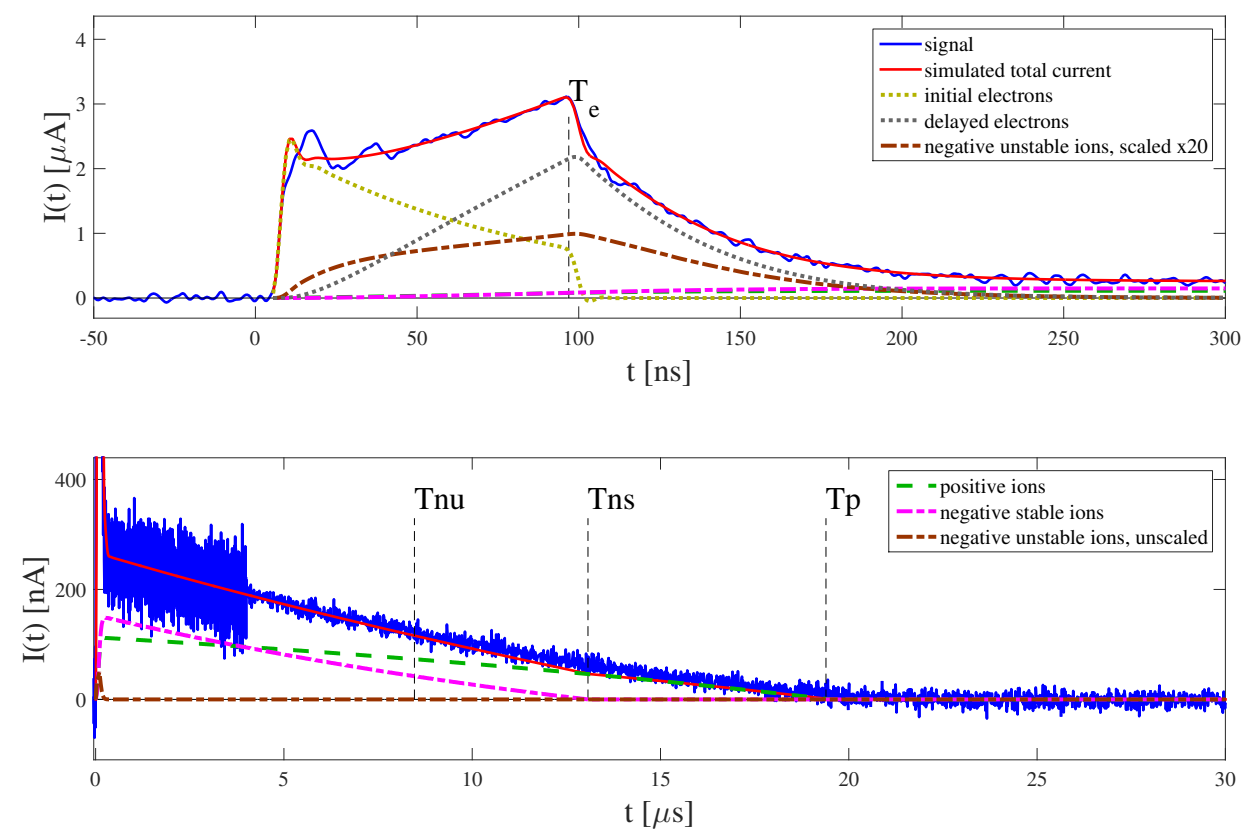

Figure 1. Measured displacement current in dry air at a pressure of $100 \mathrm{kPa}$, with electrode spacing $11 \mathrm{~mm}$ and $E / N=100 \mathrm{Td}$. The plots show an averaged signal in blue over 200 shots, on two different time-scales. In red, the result of the fitting procedure is shown. The signal in the bottom plot has been filtered, starting from $4 \mu \mathrm{s}$, for better visibility. The dashed vertical lines indicate the gap crossing time for electrons ("e"), negative unstable ("nu") ions, negative stable ("ns") ions and positive ions ("p") in accordance to the chosen model (figure 3). Furthermore, the individual contributions of different species to the current are shown in dashed and dotted lines. The attribution to specific species $\left(\mathrm{O}^{-}\right.$, $\mathrm{O}_{2}^{-}, \mathrm{O}_{3}^{-}$) is complicated and discussed in the model section. In the top plot, the current of the negative unstable ions is multiplied by 20 for better visibility.

\section{Kinetic Model}

\subsection{Review of physical processes}

A large number of processes have to be considered for the modeling of dry synthetic air (79\% nitrogen, $21 \%$ oxygen) around the $E / N_{\text {crit }}$, since the energies of free electrons are sufficient to dissociate molecules. An overview of the presumably most relevant is given in figure 2. The following review is largely based on [13, 15]. Similar kinetic schemes have been used by e.g. Zhao and Lin in $\mathrm{N}_{2}-\mathrm{O}_{2}$ mixtures [20], and by O'Neill and Craggs in oxygen [21].

Electrons of kinetic energy above the ionization threshold can create, on impact, $\mathrm{O}_{2}^{+}$ $(\approx 12 \mathrm{eV})$ and $N_{2}^{+}(\approx 15.5 \mathrm{eV})$ ions:

$$
\begin{aligned}
& \mathrm{O}_{2}+e^{-} \Rightarrow \mathrm{O}_{2}^{+}+2 e^{-} \\
& N_{2}+e^{-} \Rightarrow N_{2}^{+}+2 e^{-} .
\end{aligned}
$$

Simulations based on cross section data $[22,23]$ predict that the number of created $N_{2}^{+}$and $\mathrm{O}_{2}^{+}$is roughly $4: 6$ in air for $\mathrm{E} / \mathrm{N}=100 \mathrm{Td}$. Charge transfer from $\mathrm{N}_{2}^{+}$ions to 
Figure 2. Overview of relevant processes describing the interaction of electrons with neutral $\mathrm{N}_{2}$ and $\mathrm{O}_{2}$ molecules, at electric field strength around the critical electric field. We neglect interactions with excited molecules. For the dotted lines, literature is sparse or not available at elevated pressures and electric fields around $E / N_{\text {crit }}$.

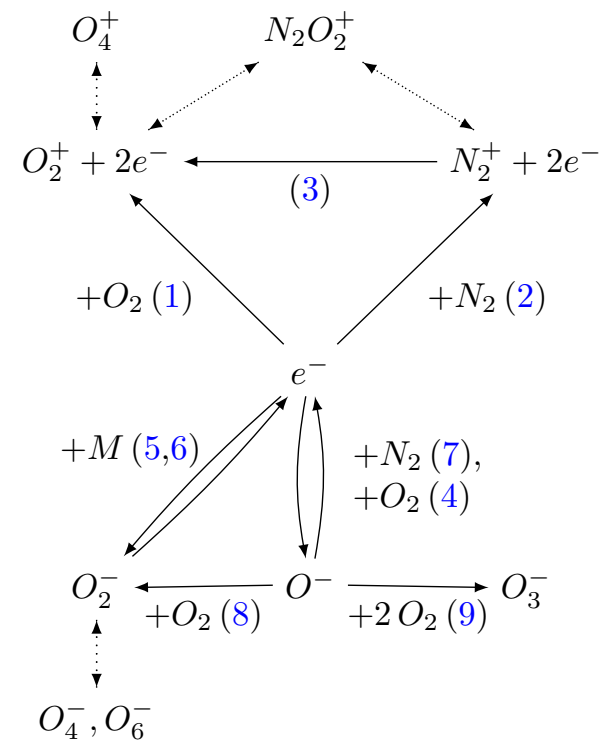

$\mathrm{O}_{2}^{+}$is assumed to be energetically favourable and very efficient at thermal ion energies without external electric field [24, 13]:

$$
\mathrm{N}_{2}^{+}+\mathrm{O}_{2} \Rightarrow \mathrm{N}_{2}+\mathrm{O}_{2}^{+} .
$$

In several experiments under near vacuum condition, formation of cluster ions like $N_{4}^{+}$and $O_{4}^{+}$was observed [24, 25, 26, 27]. Estimates of cluster decay rates without external field are given in [13]. Dissociative ionization under formation of $\mathrm{N}^{+}$and $\mathrm{O}^{+}$ requires energies above $20 \mathrm{eV}[28]$ and is unlikely at $100 \mathrm{Td}$.

Oxygen, as an electronegative molecule, exhibits attachment in two processes: the dissociative channel

$$
\mathrm{O}_{2}+e^{-} \Rightarrow \mathrm{O}^{-}+\mathrm{O},
$$

with a resonance around $6-8 \mathrm{eV}$, and direct attachment

$$
\mathrm{O}_{2}+e^{-} \Rightarrow \mathrm{O}_{2}^{-*} \stackrel{+M}{\Rightarrow} \mathrm{O}_{2},
$$

which is a three-body process at thermal electron energies. The notation of the latter process has to be understood as a capture of an electron by an oxygen molecule, resulting in an excited unstable negative ion $\mathrm{O}_{2}^{-*}$ of short life-time, which then transfers its surplus energy to a neutral molecule and stabilizes. This stabilization competes with fast (auto-)detachment of the electron. These processes are discussed in detail in [14]. At high electric fields, stabilized $O_{2}^{-}$still detaches due to collision with neutral gas molecules, but at a lower rate:

$$
\mathrm{O}_{2}^{-}+M \Rightarrow \mathrm{O}_{2}+M+e^{-} \text {. }
$$


Process (4) is expected to dominate over process (5) at electric field strength near the critical field [15]. A Bolsig+ simulation based on cross-section data [22, 28] predicts a ratio of roughly $10: 1$ at $100 \mathrm{Td}$ and atmospheric pressure.

$\mathrm{O}^{-}$ions exhibit electron detachment and two possible conversion processes:

$$
\mathrm{O}^{-}+\mathrm{N}_{2} \Rightarrow \mathrm{N}_{2} \mathrm{O}^{-*} \Rightarrow \mathrm{N}_{2} \mathrm{O}+e^{-}
$$

as considered in [7],

$$
\mathrm{O}^{-}+\mathrm{O}_{2} \Rightarrow \mathrm{O}_{3}^{-*} \Rightarrow \mathrm{O}+\mathrm{O}_{2}^{-}
$$

with subsequent detachment of the electron via (6), and

$$
\mathrm{O}^{-}+\mathrm{O}_{2} \Rightarrow \mathrm{O}_{3}^{-*} \stackrel{+M}{\Rightarrow} \mathrm{O}_{3}^{-} \text {. }
$$

The initial formation of $\mathrm{O}_{3}^{-*}$ is considered to be meta-stable [15]. The cross section for relaxation is strongly dependent on the neutral molecule $M$, and much more efficient for $\mathrm{O}_{2}$ than for $\mathrm{N}_{2}$ [14]. For the rates associated with the three-body processes (5) and (9), a quadratic dependence on (total or partial) pressure is expected.

$\mathrm{O}_{3}^{-}$is considered the sole stable negative ion in dry air. Formation of $\mathrm{O}_{4}^{-}$and $\mathrm{O}_{6}^{-}$is measured in literature in pure oxygen [29, 30, 31, 32], and cluster formation is found to occur. As with positive cluster ions, most of these experiments are performed under near-vacuum conditions and do not easily allow an extrapolation to higher pressures. In [33], the authors see evidence for $\mathrm{O}_{4}^{-}$and $\mathrm{O}_{6}^{-}$at low electric fields and pressures up to $80 \mathrm{kPa}$.

In publications concerned with plasma physics in air, excited neutral molecules are often considered important for modeling the physical behaviour [13, 34, 35, 36]. Some excited states like the oxygen singlet are supposed to be stable for nearly an hour, and not even collision with the walls de-excites those states efficiently [36]. The coefficients for interactions between electrons and excited neutral molecules, compared to the ground state, differ due to their internal energy [13]. The greatest effect would likely be expected for detachment processes, which have thresholds of few eV. In our setup around $10^{6}-10^{7}$ electrons per laser shot loose their kinetic energy in the gas volume, and are capable of creating excited neutral molecules. A rough estimation shows that we do not exceed $50 \mathrm{~nJ}$ per shot, depending on the number of released electrons and applied voltage. Assuming, as an upper estimation, $100 \%$ efficiency for the production of oxygen singlets of $0.977 \mathrm{eV}$ [37], this energy would be sufficient to excite $3 \cdot 10^{11}$ molecules per shot. This is several orders of magnitude below the particle densities at which we measure $\left(10^{24}-10^{25}\right.$ in a volume of 30 liters). We therefore neglect any distinction between ground-state and excited-state neutral molecules.

\subsection{Model}

We find that the information contained in the waveform measurements is not sufficient to deduce all rates as depicted in figure 2. Therefore, we restrict the model to a simpler approximation, which is shown in figure 3. It was originally proposed by Verhaart and van der Laan [8], and further developed by Wen and Wetzer [10, 38]. A partial analytical solution is available, however, it is unsuitable for rate fitting since some terms are only given in integral form.

Using this model we are able to fit all measurements over the entire pressure range. Based on this literature research, we assume that the relation between physical picture and this model is as follows. The ionization rate $\nu_{\mathrm{i}}$ is a superposition of the ionization of $\mathrm{O}_{2}$ and $\mathrm{N}_{2},(1)$ and (2). The positive ion is composed of $\mathrm{O}_{2}^{+}, \mathrm{N}_{2}^{+}$and potentially 
Figure 3. A common general model, first presented by Verhaart and van der Laan [8], and further developed by Wen and Wetzer [10]. The subscript of all rates $\nu$ denotes attachment ("a"), detachment ("d"), ionization ("i") and conversion ("c"). "n.s." stands for negative stable ion, "n.u." for negative unstable.

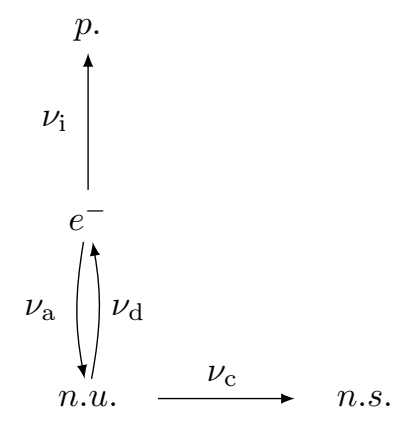

the cluster ions $\mathrm{O}_{4}^{+}$and $\mathrm{N}_{2} \mathrm{O}_{2}^{+}$. Attachment is mainly due to dissociative attachment of $\mathrm{O}_{2}$ under formation of $\mathrm{O}^{-}$(4) at electric fields around $E / N_{\text {crit }}$. We find it likely to identify the negative stable ion with $O_{3}^{-}$. The conversion rate thus $\nu_{\mathrm{c}}$ relates to the processes of three-body $\mathrm{O}_{3}^{-}$creation, (9). The role of the negative unstable ion is complicated, since charge transfer from $\mathrm{O}^{-}$to $\mathrm{O}_{2}^{-}$(8), and three body-attachment under formation of $\mathrm{O}_{2}^{-}(5)$ is not modeled. Therefore, the individual contribution of $\mathrm{O}^{-}, \mathrm{O}_{2}^{-}$and, potentially, $\mathrm{O}_{4}^{-} / \mathrm{O}_{6}^{-}$cannot be distinguished. Similarly, the detachment rate $\nu_{\mathrm{d}}$ does not relate directly to a single physical process. We report the results in the nomenclature of the model rather than the physical species, in order to reflect these uncertainties.

The corresponding system of partial differential equations for one spatial dimension, describing the charge carrier densities, reads:

$$
\begin{aligned}
& \left(\frac{\partial}{\partial t}+w_{\mathrm{dr}}^{\mathrm{e}} \frac{\partial}{\partial x}\right) \quad \rho^{\mathrm{e}}(x, t) \quad= \\
& \left(\nu_{\mathrm{i}}-\nu_{\mathrm{a}}\right) \quad \rho^{\mathrm{e}}(x, t)+\nu_{\mathrm{d}} \quad \rho^{\text {n.u. }}(x, t) \\
& \begin{array}{rllll}
\left(\frac{\partial}{\partial t}+w_{\mathrm{dr}}^{\text {n.u. }} \frac{\partial}{\partial x}\right) & \rho^{\text {n.u. }}(x, t) & = & \\
\nu_{\mathrm{a}} & \rho^{\mathrm{e}}(x, t) & -\left(\nu_{\mathrm{d}}+\nu_{\mathrm{c}}\right) & \rho^{\text {n.u. }}(x, t)
\end{array} \\
& \left(\frac{\partial}{\partial t}+w_{\mathrm{dr}}^{\mathrm{p}} \frac{\partial}{\partial x}\right) \quad \rho^{\mathrm{p} \cdot}(x, t) \quad=\nu_{\mathrm{i}} \quad \rho^{\mathrm{e}}(x, t) \\
& \left(\frac{\partial}{\partial t}+w_{\mathrm{dr}}^{\text {n.s. }} \frac{\partial}{\partial x}\right) \quad \rho^{\text {n.s. }}(x, t)=\nu_{\mathrm{c}} \quad \rho^{\text {n.u. }}(x, t)
\end{aligned}
$$

where $\rho(x, t)$ with superscripts denotes electrons and ion species densities, and $w_{\mathrm{dr}}$ the macroscopic drift velocity. The unit of all rates, $\nu_{\mathrm{i}}$ (electron ionization), $\nu_{\mathrm{a}}$ (electron attachment), $\nu_{\mathrm{d}}$ (electron detachment) and $\nu_{\mathrm{c}}$ (ion conversion), is $\mathrm{s}^{-1}$.

For simpler models without detachment, the effective electron growth rate is given as $\nu_{\mathrm{i}}-\nu_{\mathrm{a}}$. When including detachment and conversion processes, the "effective rate" becomes $[9,8,38]$ :

$$
\begin{aligned}
\nu_{\mathrm{eff} \pm}= & \frac{1}{2}\left(\nu_{\mathrm{i}}-\nu_{\mathrm{a}}-\nu_{\mathrm{d}}-\nu_{\mathrm{c}}\right) \pm \\
& \frac{1}{2} \sqrt{\left(\nu_{\mathrm{i}}-\nu_{\mathrm{a}}-\nu_{\mathrm{d}}-\nu_{\mathrm{c}}\right)^{2}+4\left(\nu_{\mathrm{i}} \nu_{\mathrm{d}}+\left(\nu_{\mathrm{i}}-\nu_{\mathrm{a}}\right) \nu_{\mathrm{c}}\right)}
\end{aligned}
$$

The analytic solution for the temporal evolution of the electron number $n_{\mathrm{e}}(t)$ for times not exceeding the electron gap drift time can be expressed as

$$
n_{\mathrm{e}}(t)=a e^{\nu_{\mathrm{eff}}+t}-b e^{\nu_{\mathrm{eff}}-t},
$$


with the pre-factors $a$ and $b$ depending on the rates. Since $\nu_{\text {eff }}>\nu_{\text {eff }-}$, the condition $\nu_{\text {eff }}:=\nu_{\text {eff }+}=0$ is sufficient to determine the critical electric field strength:

$$
\nu_{\text {eff }}=0 \Leftrightarrow \nu_{\mathrm{i}} \nu_{\mathrm{d}}+\left(\nu_{\mathrm{i}}-\nu_{\mathrm{a}}\right) \nu_{\mathrm{c}}=0
$$

It is interesting to see that in the limit of high conversion rates or low detachment rates, $\nu_{\mathrm{d}} / \nu_{\mathrm{c}} \approx 0, \nu_{\mathrm{eff}}$ reduces to $\nu_{\mathrm{i}}-\nu_{\mathrm{a}}$ :

$$
\begin{array}{ll}
\nu_{\text {eff }} \underset{\nu_{\mathrm{d}} / \nu_{\mathrm{c}} \rightarrow 0}{=} & \frac{1}{2} \nu_{\mathrm{c}}\left(\frac{\nu_{\mathrm{i}}-\nu_{\mathrm{a}}}{\nu_{\mathrm{c}}}-1\right)+ \\
& \frac{1}{2} \nu_{\mathrm{c}} \sqrt{\left(\frac{\nu_{\mathrm{i}}-\nu_{\mathrm{a}}}{\nu_{\mathrm{c}}}-1\right)^{2}+4 \frac{\nu_{\mathrm{i}}-\nu_{\mathrm{a}}}{\nu_{\mathrm{c}}}} \\
= & \nu_{\mathrm{i}}-\nu_{\mathrm{a}}
\end{array}
$$

As initial condition for the simulation, the densities are set to zero except for an electron peak at $x=0$. Based on the simulation of charge carrier densities, the resulting displacement current is calculated, and compared with the measured signal. Starting with randomly chosen rates, the optimization routine tries to improve on the initial guess and minimize the difference between signal and simulation. The preset range for the initial starting point covers $1-2$ order of magnitude for all rates. Within these boundaries, the fit almost always converges to the same results, within a certain spread. We therefore assume that the solution is unique. Details about the simulation and fitting procedure can be found in the appendix.

Figure 1 shows, as an example, the individual contributions to the current as derived in the fitting procedure. The yellow dotted line shows the current contribution of the initial electron pulse. It is interesting to note that the attachment rate coefficient is higher than the ionization rate coefficient at a density-reduced electric field of $100 \mathrm{Td}$ in air, while the total current seems to grow exponentially until $100 \mathrm{ns.} \mathrm{In} \mathrm{dotted} \mathrm{grey,}$ "delayed electrons" which have been captured by a neutral molecule and subsequently detach are shown. A significant number of unstable ions (dot-dashed brown) are present only for a limited time, due to conversion and detachment processes.

\section{Results}

The chamber temperature was stable at $295.5 \mathrm{~K}$, and we calculate the particle density $N$ using the ideal gas law. We report ion mobilities as density-normalized $\mu \mathrm{N}$ in units of $\mathrm{s}^{-1} \mathrm{~m}^{-1} \mathrm{~V}^{-1}$ such that the velocity at measurement temperature is given as $\mu \mathrm{N} \cdot \mathrm{E} / \mathrm{N}$. The following parameters are preset and not fitted:

- We estimate the density-normalized longitudinal electron diffusion coefficient as roughly $N D_{\mathrm{L}}=2 \cdot 10^{24} \mathrm{~s}^{-1} \mathrm{~m}^{-1}$ with a rather large uncertainty. Apart from the initial electron-dominated current, diffusion barely influences the current shape. Ion diffusion is neglected.

- The mobility of the positive ions can be estimated by matching the latest current of the measurement with the simulation (see figure 1, after $15 \mu \mathrm{s}$ ). The current is strongest for the highest measured $\mathrm{E} / \mathrm{N}$ and highest pressures, from which we read off a density-normalized ion mobility of $\mu \mathrm{N}=5.6 \cdot 10^{21} \mathrm{~s}^{-1} \mathrm{~m}^{-1} \mathrm{~V}^{-1}$. Comparing to the reported mobility of $\mathrm{O}_{2}^{+}$in air, this is $17 \%$ lower than values from [39] and data from LXCat $[40,22]$, which agree on a density-normalized mobility of $\mu \mathrm{N} \approx 6.8 \cdot 10^{21} \mathrm{~s}^{-1} \mathrm{~m}^{-1} \mathrm{~V}^{-1}$ at $E / N=100 \mathrm{Td}$. The lower mobility of the positive ion could hint at ion cluster formation (see section 3 ), since ion mobilities usually decrease with growing cluster radius. The latter has e.g. been discussed for pure oxygen in drift-tube experiments [41]. 
- For the negative unstable and stable ion we use literature values from [39] for $\mathrm{O}^{-}$ and $\mathrm{O}_{3}^{-}$, respectively. As we discussed in the model section, the role of further negative ions $\left(\mathrm{O}_{2}^{-}\right.$and $\left.\mathrm{O}_{4}^{-}\right)$is not entirely clear, and we might thus make a certain error in the mobilities of the negative ions.

The time-points of the raising and falling edge of the measurements contain information about the drift velocity of the electrons, which relates to the density-reduced mobility as $w=\mu^{\mathrm{e}} N \cdot E / N$. Our fitted values match well with electron mobilities reported by Frommhold [4] (see figure 4) and Bolsig+ simulation using Biagi's database $[28,22]$.

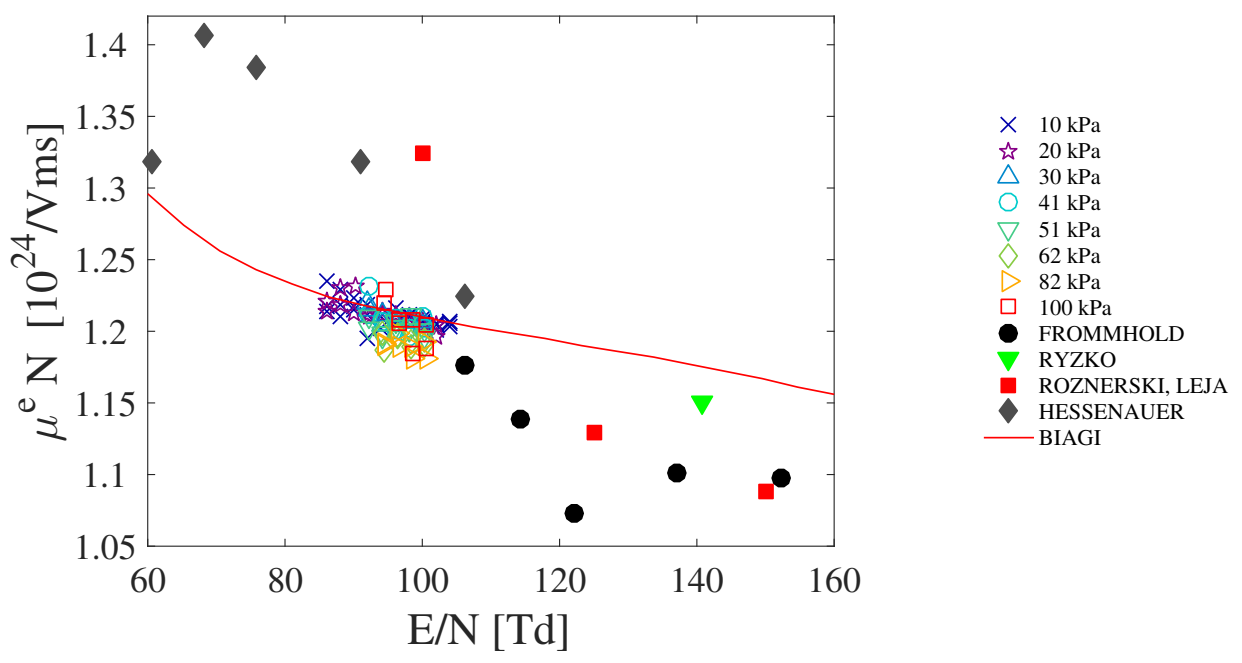

Figure 4. Reduced electron mobility $\mu^{\mathrm{e}} N$, compared to data from [4, 42], both downloaded from [22], and [43, 44] as well as Bolsig + simulation using Biagi's database.

The results for the rate coefficients are shown in figures 5 to 9 . For comparison we have included, where available, results from previous Townsend and drift tube experiments, as well as results from Bolsig + simulations using cross sections from the LXCat database [22]. The models used by Frommhold [4], Prasad [6], Harrison and Geballe [5], and Ryzko [46] are different from ours, and do not include ion conversion. Wagner [45] uses the same model, but interprets the stable negative ion differently. The Bolsig + simulations have been compared to more complex Monte-Carlo simulations and were found to agree reasonably well in the energy range considered in this publication $[47,48]$. In cases where electron velocities are needed for converting units and not provided by the author, we use reference data from [4].

In the chosen model we have not distinguished between the individual ionization rates of $\mathrm{N}_{2}$ and $\mathrm{O}_{2}$. The information contained in the measured current traces is not sufficient to separate both rates individually, whereas the sum of both is reliably extractable. The ionization rate coefficient, figure 5, increases strongly with increasing $E / N$ in the measurement range of $86-104 \mathrm{Td}$. The spread in the rate coefficient decreases with increasing pressure. The reason is that measurements at higher pressures exhibit a higher ion current, which benefits the signal-to-noise ratio. Experimental data from $[6,5,45]$ agree qualitatively with our findings, but show a larger scatter 


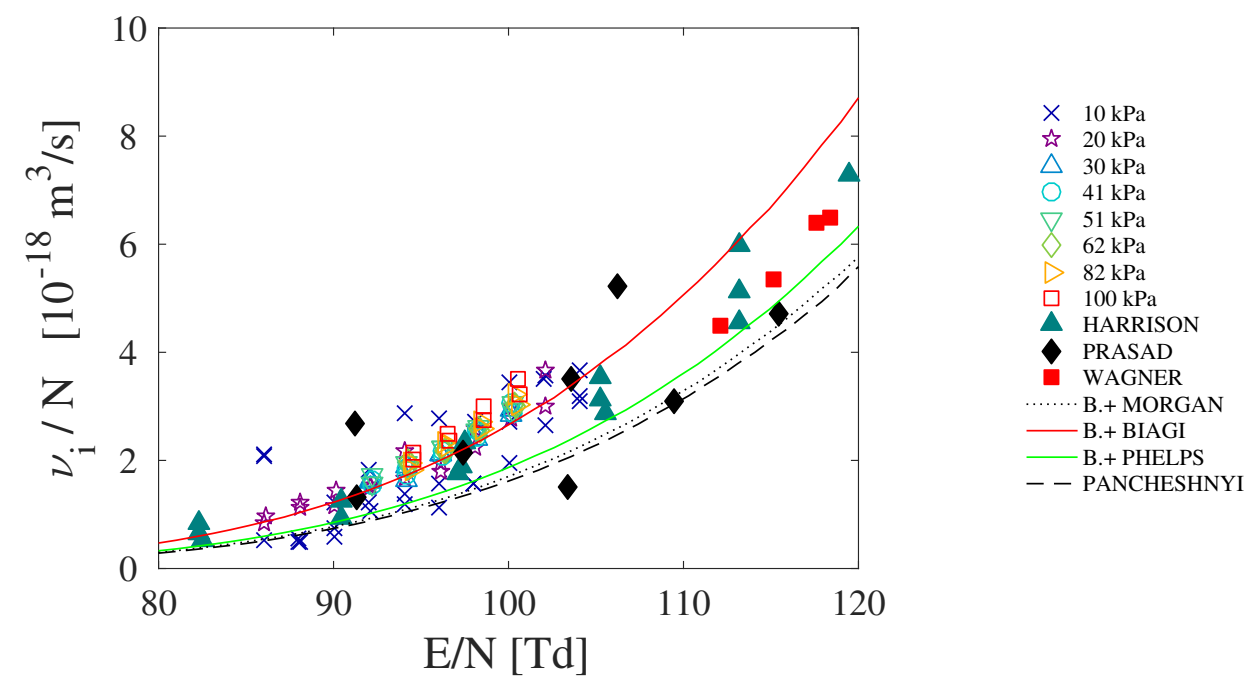

Figure 5. The (total) ionization rate coefficient of synthetic air is plotted against $E / N_{\text {crit }}$. We measure a superposition of $\mathrm{O}_{2}$ and $N_{2}$ ionization, which cannot be separated. The results from several Bolsig + simulations, based on tabled crosssections (source: LXCat [22]) are plotted for comparison. The accordance is especially good for the Biagi database. References are taken from $[6,15,45,5]$.

and are lower on average. We assume that the differences in the used models in these publications have a minor influence on the results for the ionization rate coefficient, so that comparability is given. Furthermore, Bolsig + simulations based on different databases are plotted. Using Biagi's cross section set matches our findings closest. Overall, the shape and order of magnitude of our results are in good agreement to literature values, considering the large scatter in these data reported by different authors.

The attachment rate coefficient $\nu_{\mathrm{a}} / N$ is plotted in figure 6 . Our findings and all references agree that the rate coefficient increases with increasing electric field. This is explained by a shift in the electron energy distribution towards higher energies, which then overlaps to a larger extent with the dissociative attachment resonance at $6-8 \mathrm{eV}$. Older measurements differ by up to a factor of 4 around $110 \mathrm{Td}$. Our findings match those of Frommhold [4] closest, while Prasad [6], Harrison and Geballe [5] and Ryzko [46] have measured lower values and Wagner higher values. The cited reference data were obtained using different models and experimental setups, which might explain part of the discrepancy.

Several databases for Bolsig + simulations feature cross sections for both dissociative attachment and, additionally, direct three-body attachment to $\mathrm{O}_{2}$ (equation (5)). The latter is to be scaled according to (partial) oxygen pressure. We plot both the dissociative attachment rate coefficient alone (zero pressure limit), and the sum of three- and two-body attachment rate coefficients, scaled to $100 \mathrm{kPa}$. The difference is small for all databases $(\approx 5-10 \%)$, in agreement with our assumptions in section 3. For the Biagi database we took the three-body attachment cross-sections from the Magboltz source (version 11.2) [28], which differ strongly from the older version (8.97, transcripted in 2012) available on LXCat.

Our attachment rate coefficient shows a certain pressure dependency, which amounts 


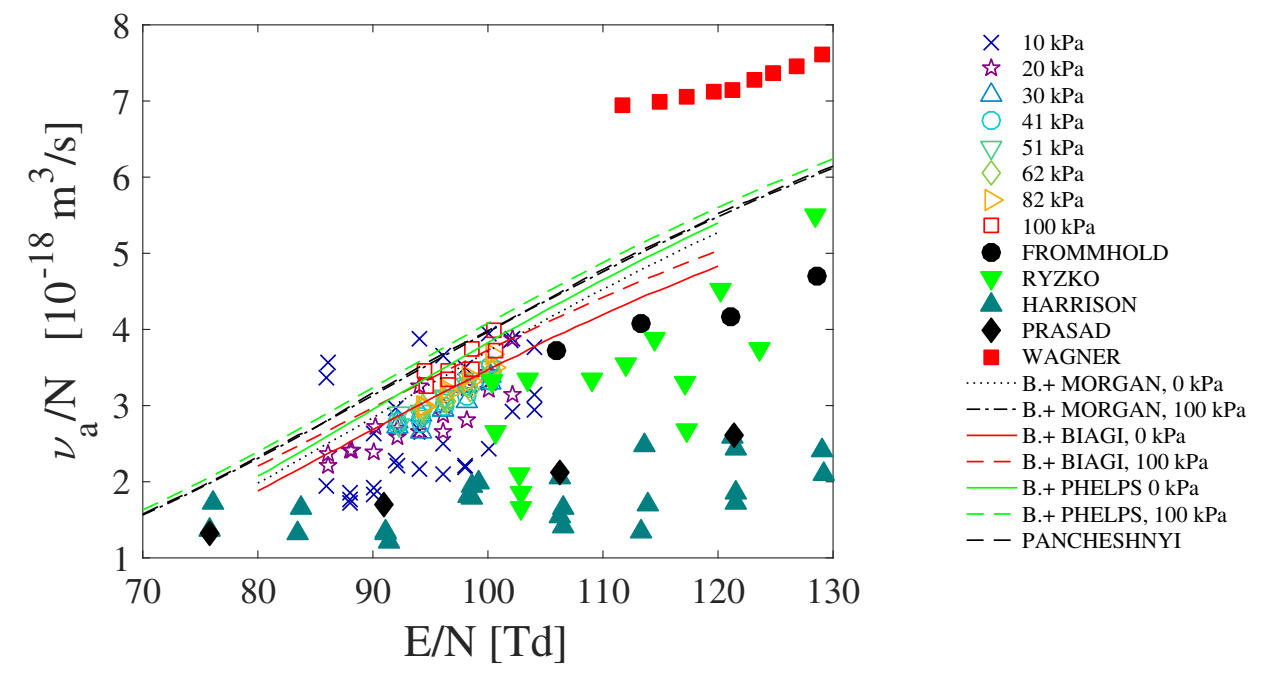

Figure 6. The attachment rate coefficient of synthetic air is plotted against $E / N_{\text {crit }}$. Various references from measurements are plotted for comparison, as well as Bolsig + simulation results. Cross-sections are available for both dissociative attachment (as in our model), as well as three-body non-dissociative attachment to $\mathrm{O}_{2}$. For Bolsig + simulations annotated with " $100 \mathrm{kPa}$ " both contributions are summed up. References are taken from [4, 6, 5, 45, 46, 15].

to about $20 \%$ difference on average between the findings for $10 \mathrm{kPa}$ and $100 \mathrm{kPa}$. To a smaller extent, a pressure dependency is also visible for the ionization and conversion coefficient. Overall, the findings agree very well with Bolsig + calculations using Phelps, Morgan and Biagi databases.

The detachment rate coefficient is plotted in figure 7 . There are experimental results available from Wagner [45], Frommhold [3], and Ryzko [46], who have measured mainly at higher $E / N$. The models used in these references are comparable to the present model. Pancheshnyi's theoretical calculations for $\mathrm{O}^{-}$conversion to $\mathrm{N}_{2} \mathrm{O}^{-*}$ and $O_{2}^{*}$ are given in the figure.

The conversion rate coefficient is plotted in figure 8 as rate per second over particle density squared, since three-body processes are expected to be quadratic in density. Again, the spread of our findings decreases drastically with increasing pressure. The rate's quadratic proportionality on pressure can clearly be demonstrated: The rates $\nu_{\mathrm{c}}$ in units $s^{-1}$ that are fitted are about a factor of 100 higher for the $100 \mathrm{kPa}$ measurements compared to $10 \mathrm{kPa}$. References for this process are sparse in literature. Pancheshnyi provides a theoretical approximation [15], which is based on rates in cold air plasma without external electrical field (Kossyi [13]). For this reference we assume that the rate should be scaled with total pressure as in the publication of Kossyi, and not partial oxygen pressure (see also equation (9)). There are measurements available from Wagner [45], where the same model is used as in this publication. However, the physical processes are interpreted differently, $\mathrm{O}_{3}^{-}$is considered negligible and $\mathrm{O}_{2}^{-}$ takes the role as the sole stable negative ion. As far as the accuracy allows, Wagner reports the conversion processes as linear in particle density. Accordingly, they differ 


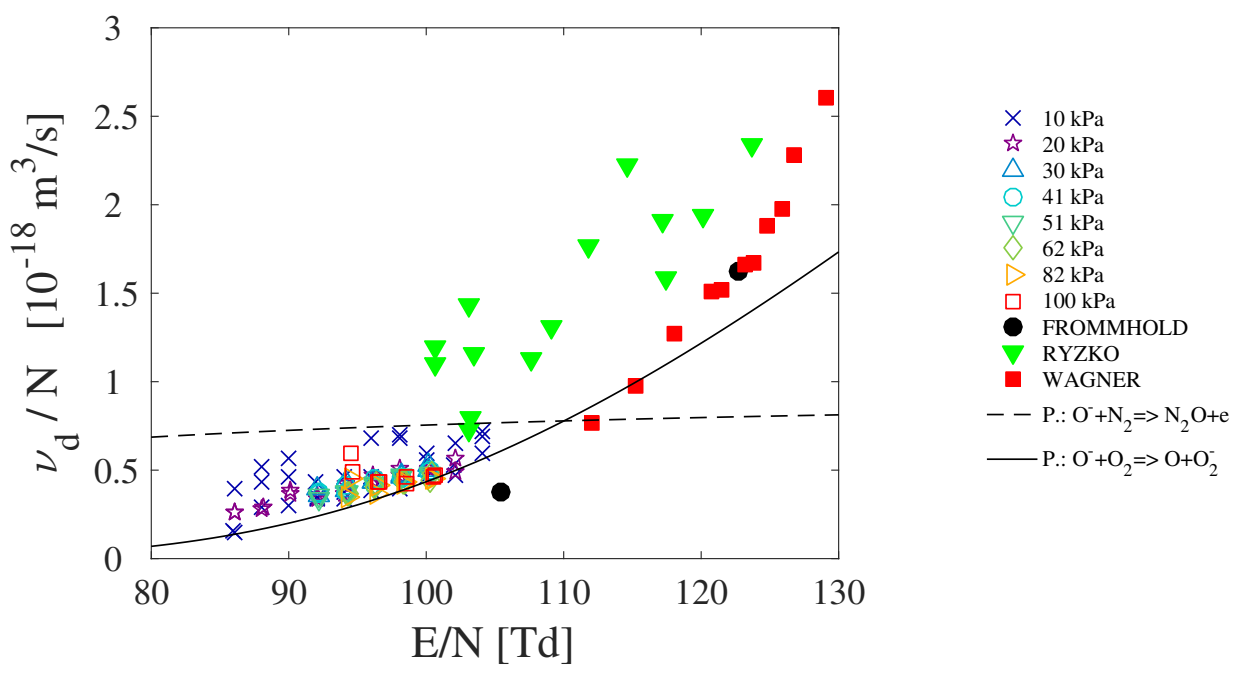

Figure 7. The plot shows the detachment rate coefficient in synthetic air as introduced in the model section. Measurements with similar models are available from Frommhold, Wagner, and Ryzko. Furthermore, theoretical calculations from Pancheshnyi (abbreviated as "P.") for $\mathrm{O}^{-}$conversion to $\mathrm{N}_{2} \mathrm{O}^{-*}$ and $\mathrm{O}_{2}^{-}$, both which are followed by electron detachment, are plotted. References are taken from $[4,45,46,15]$.
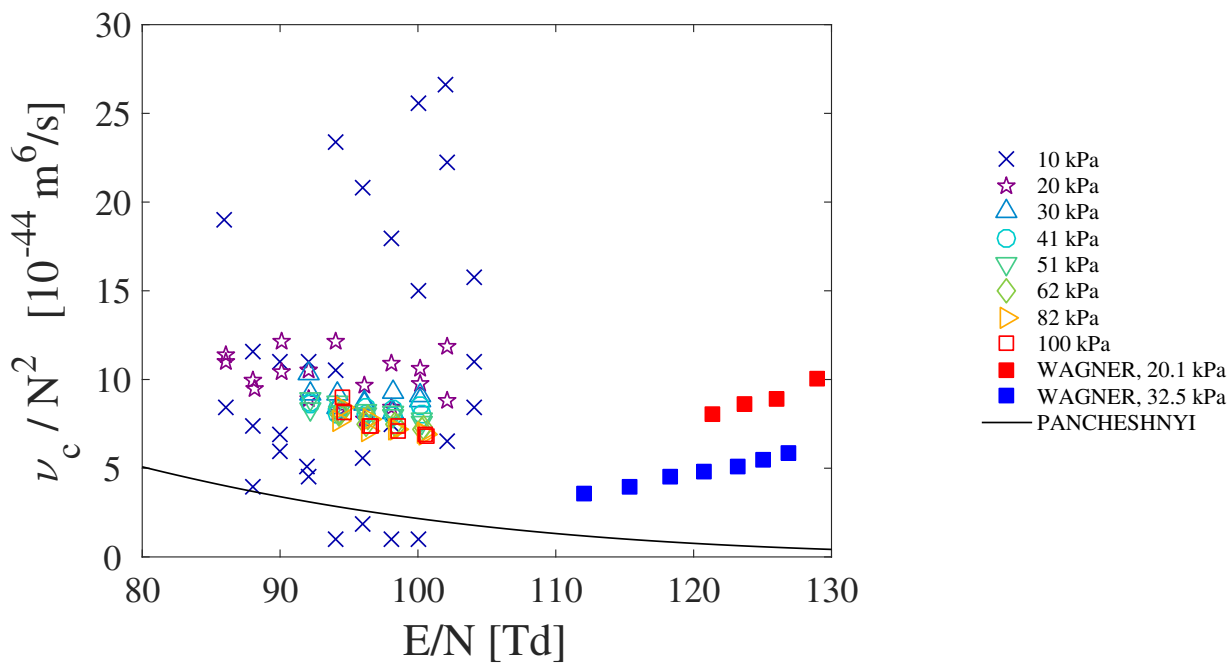

Figure 8. The conversion rate coefficient in synthetic air relates to the process of $\mathrm{O}_{3}^{-}$formation, equation (9). It is plotted as rate over density squared. References are taken from [15, 45].

when plotted as rate over density squared. Compared to our results the magnitude of the findings agrees.

Finally, in figure 9 on the left the effective rate coefficient is plotted for different pressures. It is calculated from the fitted rates according to equation (11). For each 

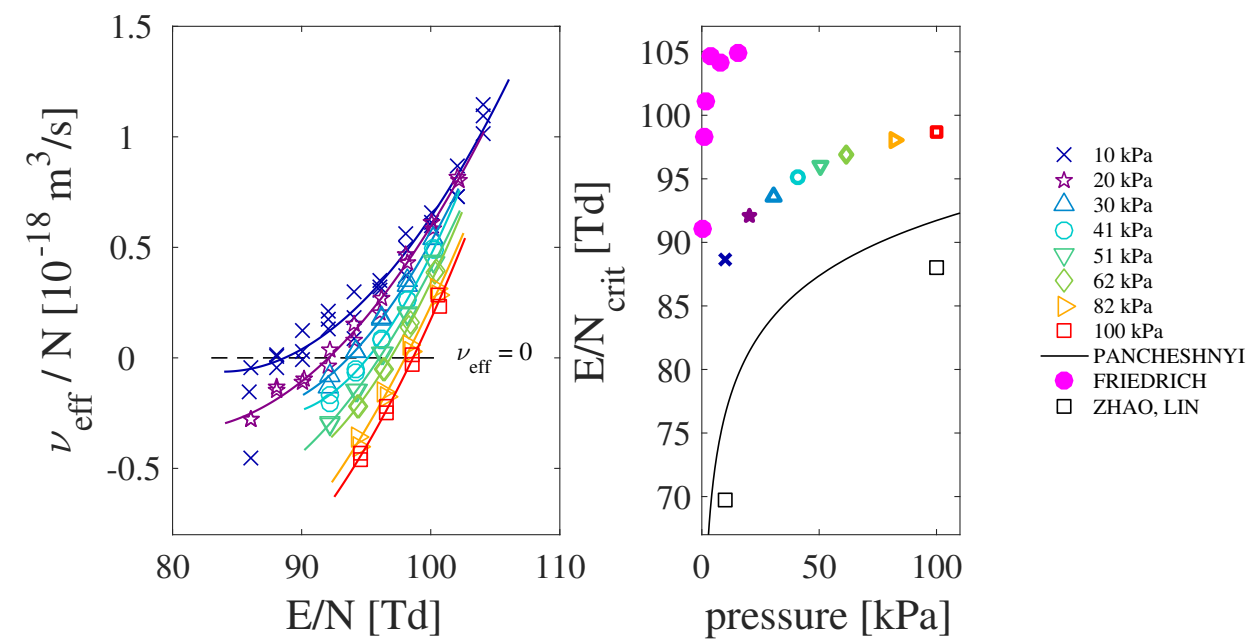

Figure 9. The left plot shows the effective rate coefficient, calculated from the fitted rates $\nu_{\mathrm{i}}, \nu_{\mathrm{a}}, \nu_{\mathrm{d}}, \nu_{\mathrm{c}}$ according to equation (11). Since the rate $\nu_{\mathrm{c}}$ is quadratic in pressure, a pressure dependency of the effective rate is expected (see 11). The right plot shows an estimate of the critical field strength according to equation (13), estimated via a quadratic fit to obtain the (pressure-dependent) zero-crossing of the effective rate as defined. The value is plotted against the respective pressure values of our measurement. The theoretical predictions of $[15,20]$ are plotted as reference, as well as measurements of Friedrich [12].

subset of data points at equal pressure, the effective rate coefficient is strongly increasing with increasing $E / N$. On the right side, $E / N_{\text {crit }}$ is plotted against pressure. It is obtained by a (quadratic) polynomial least-square fit, as indicated by the solid lines in the left plot of figure 9. The results are compared to theoretical calculations of Pancheshnyi [15] and Zhao, Lin [20]. Based on tabulated cross sections, the authors predicts a $E / N_{\text {crit }}$ as shown on the right side of figure 9 . We find a roughly $8 \mathrm{Td}$ higher critical field strength, yet matching in shape compared to Pancheshnyi's results. Zhao and Lin calculate lower values for pressures below $100 \mathrm{kPa}$, which increase slower with increasing pressure. Friedrich measures an increase in $E / N_{\text {crit }}$ [12], yet increasing steeper and higher in value.

\section{Discussion}

We find that the recorded current of the Pulsed Townsend experiment contains enough information to deduce the four rate coefficients of the discussed model, including ion detachment and conversion. The spread in the rate coefficients decreases for higher pressure measurements, which is explained by higher current from ions, which benefits the signal-to-noise ratio.

From the shape of the high-pressure measurements we are able to estimate the mobility of the positive ion. Our value is not compatible with reference data for $\mathrm{O}_{2}^{+}$in air, and is approximately $17 \%$ lower. This leads us to believe that either the charge transfer of $\mathrm{N}_{2}^{+}$to $\mathrm{O}_{2}^{+}$, equation (3), is less efficient than assumed, or that the formation of heavier, slower ion clusters is non-negligible.

The sensitivity of the results with respect to the fixed input parameters diffusion, 
digital filter and ion mobilities is difficult to quantify. In our experience, diffusion and filter have a very limited influence. Changes in the mobilities of the ions, on the other hand, affect the resulting rate coefficients. A shift in mobility for the positive ion and the negative stable ion of up to $10 \%$ yields a fit that is still satisfactory and shifts the rate coefficients by up to $10 \%$. Even greater deviations in the mobility begin to degrade the fit quality, and deviations are visible in the late, ion-dominated current. The mobility of the negative unstable ion has a negligible influence, due to its short life-time. Furthermore, we see a slight pressure dependence in the rate coefficients. At least for the ionization rate coefficient a pressure dependency is hardly physically plausible. It is strongest for the attachment rate coefficient and amounts to roughly $20 \%$. The reason for this might be the discussed model insufficiency, since three-body attachment to $\mathrm{O}_{2}$ was not taken into account. Another possible source is a pressure dependent deviation in the electrode distance, since our method of correction has a finite accuracy (see section 2).

The ionization rate coefficient could be deduced at a higher accuracy than in previous reported measurements. It fits particularly well with Bolsig + calculations based on Biagi's database.

Reference data from different experiments and models do not agree well for the attachment rate coefficient, as far as they are comparable. As for the ionization rate coefficient, our findings match with Bolsig + calculations.

For the chosen model, the detachment rate coefficient does not relate to a single process, but is influenced by detachment of $\mathrm{O}^{-}$and $\mathrm{O}_{2}^{-}$, direct attachment to $\mathrm{O}_{2}$ and conversion between the two ions (equations (4)-(9)). It is therefore not easily comparable to theoretical calculations.

The conversion rate coefficient relates to the three-body attachment process $\mathrm{O}^{-}+$ $2 \mathrm{O}_{2} \rightarrow \mathrm{O}_{3}^{-}+\mathrm{O}_{2}$, equation (9). Compared to the theoretical value of Pancheshnyi [15], we find a higher rate coefficient by a factor of $2-3$.

Our findings for the critical field strength match in shape with the calculations of the same author, yet with an offset of $8 \mathrm{Td}$. Zhao and Lin's publication [20] is similar to Pancheshnyi's, who both use a Boltzmann solver to calculate rate coefficients. They predict a much slower increase in $E / N_{\text {crit }}$ with pressure. Friedrich's experimental results [12], using a Pulsed Townsend experiment, show a much faster increase in $E / N_{\text {crit }}$, converging to $\approx 104 \mathrm{Td}$. The reason for the pronounced difference in the pressure dependency is unclear.

With the conversion rate being quadratic in pressure, in contrast to attachment, ionization and detachment rate being linear in pressure, the observed pressure dependency of the effective rate becomes clear. With equation (14), one can now attempt to predict the behaviour at application pressures of several $100 \mathrm{kPa}$. Threebody attachment to $\mathrm{O}_{2}$ might not be negligible at these pressures, which would increase the critical field strength. Therefore only a lower bound for $E / N_{\text {crit }}$ of dry synthetic air can be predicted as $105 \mathrm{Td}$ at room temperature.

\section{Summary and Outlook}

In this work we have derived and presented our method of rate fitting, and measured a pressure-dependent critical density-reduced field strength in dry air. Our findings show good agreement with experimental references where available. Theoretical predictions match for electron ionization and attachment, but differ for the ion conversion process. 
We have further demonstrated the need to measure at elevated pressures above few $10 \mathrm{kPa}$ in dry air: the conversion rate coefficient, in particular, could not be reliably determined from our low-pressure measurements.

\section{Acknowledgments}

This work is financially supported by GE Grid (Switzerland) GmbH, ABB Switzerland, Pfiffner Technologie AG, Siemens AG.

\section{Appendix A. Simulation}

The simulation of waveforms for a given set of rates and drift times is by far the most time consuming step in the optimization procedure. Dealing with rates with frequencies up to $10^{9} \mathrm{~s}^{-1}$, and orders of magnitude difference between highest and lowest (non-negligible) rates, poses a challenge for the numerical evaluation. The need to resolve the time-dependent spatial distribution of electrons, enforces a timestep $\mathrm{d} t$ below $10^{-9} \mathrm{~s}$. On the other hand, the different time-scales of ions compared to electrons (typically factor $\approx 100$ ) lead to time consuming simulations when trying to use the whole information of the late ion-dominated current, with a typical simulation length of $2 \cdot 10^{-5} \mathrm{~s}$. Due to detachment processes, a substantial amount of free electrons are present over the whole simulation time, ruling out an increase of the time-step after e.g. the electron crossing time.

We implement the simulation in $\mathrm{C}++$ and CUDA $\mathrm{C}$, and use a matlab mex / mexcuda interface function in order to minimize computation time. Two GTX 1080 cards are used for the simulation. The setup is capable of simulating the full current up to $5 \cdot 10^{-5}$ s sufficiently fast: 160 waveforms simultaneously in few tenths of a second, for the described model. Compared to our CPU implementation, the speedup is substantial.

The space between cathode and anode is represented by $N_{\mathrm{x}}$ equally spaced cells, and is described by the density of each species in those cells. The current at (discrete) time $t$ is then proportional to

$$
I(t)=\sum_{j=1}^{N_{\mathrm{S}}} \sum_{i=1}^{N_{\mathrm{x}}} e \rho^{j}\left(x_{i}, t\right)\left|w_{\mathrm{dr}}^{j}\right|
$$

with electron charge $e$, number of species $N_{\mathrm{S}}$, total number of cells $N_{\mathrm{x}}$, drift velocity $w_{\mathrm{dr}}^{j}$ of species $j$, and the number of species $j$ in cell $i, \rho^{j}\left(x_{i}, t\right)$ ("densities").

In every time-step, $\vec{\rho}$ is updated according to equation (10), which we write for simplicity in matrix form

$$
\begin{array}{ll}
\left(\frac{\partial}{\partial t}+\vec{w} \frac{\partial}{\partial x}\right) \vec{\rho}(t)=M \vec{\rho}(t) & \text { with } \\
\vec{\rho}(t)=\left[\rho^{\mathrm{e}}(t), \rho^{O_{2}^{+}}(t), \rho^{N_{2}^{+}}(t), \ldots\right]^{\mathrm{T}} . &
\end{array}
$$

Matrix $M$ contains the rates of equation (10). The effective rate of equation (11) then identifies as the largest non-zero eigenvalue of $M$. In every time-step, the update of densities is followed by advection of species. Since the macroscopic drift velocity of species $j$ (electron/ion) is constant in the homogeneous field, the transport terms (second term of l.h.s. of equations (A.2) and (10)) can be simplified such that a full 
translation from one cell to the next is executed within $d /\left(N_{x} w_{\mathrm{dr}}^{j}\right)$, with electrode gap distance $d$. We set the time-step of the simulation as

$$
\mathrm{d} t=\frac{d}{N_{x} w_{\mathrm{dr}}^{\mathrm{e}}},
$$

such that electrons are translated every time-step, and ions every $\approx 100$ time-steps.

A direct simulation via a suitable Runge-Kutta time-stepping method is possible, but very resource-demanding. We experience that the time-step has to be reduced drastically for higher rates in order to achieve convergence.

A direct numerical evaluation of the r.h.s. of equation (A.2) is challenging, due to the numerical properties of matrix $M$ : it is often badly conditioned, exhibiting orders of magnitude difference between (non-zero) eigenvalues. Matlab offers with the builtin function "expm" an implementation of the Pad approximation [49] for the direct evaluation of the exponential, which is used in the following.

For the implementation we want to specifically point out two things that increased the numerical stability when using single precision floats:

- The matrix $e^{M \mathrm{~d} t}$, equation (A.2), approaches, for $\mathrm{d} t \Rightarrow 0$, the unity matrix. In our case it was useful to store and apply $e^{M \mathrm{~d} t}-\mathbb{1}$ in order to preserve the accuracy better.

- In every time-step, the densities are updated as $\vec{\rho} \leftarrow \vec{\rho}+e^{M \mathrm{~d} t} \vec{\rho}$. Direct implementation leads to an accumulation of error due to the numerically unfavorable summation of numbers of very different magnitude. It is more stable to introduce a temporary density vector, and add up the change in density for several time-steps, before updating.

\section{Appendix A.1. Diffusion}

Incorporation of longitudinal spatial diffusion of electrons is of importance when comparing signal and simulation at times around the electron drift time. Diffusion of ions is considered less significant and is disregarded. In the systems of equations (10), we add a diffusion term on the right-hand-side of the form

$$
\left(\frac{\partial}{\partial t}+w_{\mathrm{dr}}^{\mathrm{e}} \frac{\partial}{\partial x}\right) \rho^{\mathrm{e}}(x, t)=\cdots-D \frac{\partial^{2}}{\partial x^{2}} \rho^{\mathrm{e}}(x, t) .
$$

Numerically, we define a diffusion operation $\hat{\rho}^{\mathrm{e}}=\Phi_{A}\left[\rho^{\mathrm{e}}\right]$ of strength $A$ as

$$
\begin{aligned}
& \hat{\rho}^{\mathrm{e}}\left(x_{i}, t\right)=\rho^{\mathrm{e}}\left(x_{i}, t\right)-\frac{A \mathrm{~d} t}{\mathrm{~d} x^{2}} \times
\end{aligned}
$$

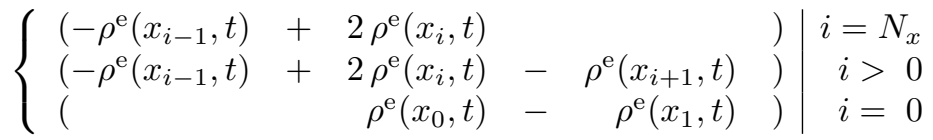

where the first cell nearest to the cathode is treated separately as an impermeable boundary. This prevents an artificial "loss" of electrons, when diffused backwards; especially at the beginning of the simulation.

Equation (A.5) is a valid approximation only if $\frac{A \mathrm{~d} t}{\mathrm{~d} x^{2}} \ll 1$, and we therefore approximate equation (A.4) as a loop over $n$ diffusion operations with $A=D / n$ :

$$
\rho^{\mathrm{e}}=\Phi_{D / n}\left[\Phi_{D / n}\left[\cdots \Phi_{D / n}\left[\rho^{\mathrm{e}}\right] \cdots\right]\right]
$$

Since diffusion is a costly operation, especially on GPUs, we set $n$ as the minimum number that fulfills $\frac{A \mathrm{~d} t}{\mathrm{~d} x^{2}} \leq 0.1$. Furthermore, application of diffusion is limited to 
times before $2 T^{\mathrm{e}}$ (drift time of electrons). This approximation seems justified since the densities of electrons is both much lower and spatially smoother than the delta-pulse shaped initial condition.

\section{Appendix A.2. Filtering}

For comparison with measurement data, we apply a digital low-pass filter according to the specifications of the preamplifier onto the simulated waveform. The manufacturer supplied a phase response on request, according to which we adapt a digital Butterworth filter.

\section{Appendix A.3. Testing and Convergence}

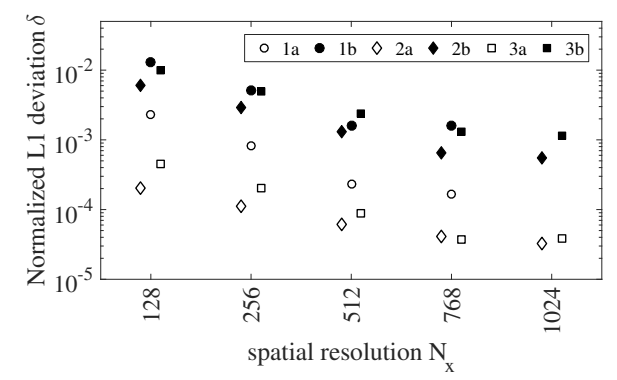

Figure A1. This plot shows the results of the convergence tests for different spatial resolutions $N_{x}$ and 5000 randomly drawn parameter sets. We plot both average (empty markers) and "worst occurrence" (filled markers) of the L1 norm distance, according to equation (A.7), for different comparisons:

- Circle: low-resolution simulation $\left(N_{x}=128 / 256 / 512 / 768\right)$ compared to high-resolution $\left(N_{x}=1024\right)$

- Diamond: simulated electron displacement current compared to analytic formula

- Square: simulated negative unstable ion displacement current compared to analytic formula

We have mentioned some of the numerical challenges of the simulation, which arise from the very different time-scales, and the order of magnitude difference in the rates. Consistency and convergence tests have been helpful for finding flaws in the implementation and numerical instabilities.

In [38], a partial analytical solution for the model at hand is given, against which the simulation can be compared. The spatial densities of electrons and negative unstable ions (yet not positive and stable negative ions) can be calculated for any time-point. In order to compare, we calculate the densities at a time-point $t$ on a spatial grid of 1000 cells. Summation over all cells, similar to equation (A.1), yields the displacement current of electrons and negative unstable ions. Unfortunately, we experience that our straight-forward implementation of the analytic expression is not stable for certain parameter sets. We therefore check its grid-convergence for 1000 and 2000 spatial grid points, and discard the result if the relative deviation is larger than $10^{-4}$. For these cases, the simulations of different spatial distribution are only compared with each other.

For all parameters of the simulation, we define suitable bounds. The upper boundary for all rates is set to $10^{9} \mathrm{~s}^{-1}$, and is more than one magnitude larger than what we fit 
in the air measurements.

For the test we randomly draw 5000 combinations of parameters. The ionization is adjusted so that the set of parameters is close to the critical field strength. We exclude parameter sets for which the late ion-dominated current exceeds the ten-fold of the initial electron current. All waveform simulations are interpolated linearly with $1 \mathrm{~ns}$ time-step, similar to like it is done in the fitting procedure. The obtained waveforms are compared using the following normalized L1 deviation:

$$
\delta=\frac{\sum_{n}\left|I_{\text {analytic } / \text { reference }}^{\mathrm{e}}\left(t_{n}\right)-I^{\mathrm{e}}\left(t_{n}\right)\right| \mathrm{d} t}{\sum_{n^{\prime}} I_{\text {analytic } / \text { reference }}\left(t_{n}\right) \mathrm{d} t} .
$$

As expected, we find improved convergence for higher spatial resolution (figure A1). An earlier attempt with Runge-Kutta fourth-order time-stepping performed much worse in this regard. There are a few occurrences of higher error, as depicted by the filled markers. The worst cases are usually "exotic" parameter sets with rates of several orders of magnitude difference, featuring steep decline of electron current or massive ion currents. We expect a less critical behaviour for "normal" experimental waveforms. We set $N_{x}=512$ for all simulations.

\section{Appendix B. Fitting process}

In the fitting procedure we try to find a set of parameters for which the quadratic norm between measurement and simulated waveform is minimal. For the optimization, we set the objective value $\xi$ as the sum over the quadratic difference between measurement $f_{t}$ and simulation $g_{t}$ :

$$
\xi=\int_{t=T_{0}}^{T}\left|f(t)-F_{\mathrm{LP}}[g(t)]\right|^{2} \omega(t) \mathrm{d} t,
$$

Here, $f(t)$ and $g(t)$ are interpolations of the time-discrete measured data and simulation, respectively. $g_{t}=g_{t}\left(\nu_{\mathrm{i}}, \nu_{\mathrm{a}}, \cdots, T^{\mathrm{e}}, T^{\mathrm{p}}, \cdots, D, n_{0}\right)$ is a function of rates $\nu$, transit times $T$, diffusion $D$ and start electron number $n_{0}$. We introduce a weighting function $\omega(t)$ and weigh the initial $2 T^{\mathrm{e}} \approx 1 \mu \mathrm{s}$ by a factor of 10 stronger than the subsequent ion-dominated current. Due to spurious oscillations at the onset of the measurement we set the lower integration limit $T_{0}$ to $30 \mathrm{~ns}$. The maximum simulation time $T$ is set to $2 \cdot 10^{-5} \mathrm{~s} . F_{\mathrm{LP}}$ is a digital low-pass filter, simulating the limited bandwidth and phase shift of the measurement system, as described in chapter 2 . The integral is summed up with a time-step $\Delta=1 \mathrm{~ns}$.

For optimization algorithms it is often beneficial to scale fitting parameters. We do so by taking the logarithm of all rates, which then lie in a range of 10 to 20 .

We use the Matlab optimization toolbox for the fitting process. The "GlobalSearch" algorithm randomly generates starting points, subject to given bounds. We choose these boundary intervals for the rates at least one order of magnitudes around the expected rate. Only those starting points are run which pass certain tests (closeness to previously found solutions, initial objective function value, ...). For any starting point that is chosen to be run, a "local solver" is called, which runs until convergence in the sense of falling below a certain threshold in the derivativeof $\xi /$ step length. For this local solver, the Matlab interior-point algorithm showed the fastest convergence. We found it necessary to control and increase the step-size of the gradient function, in order to ensure gradient smoothness. 


\section{References}

[1] A Winter, J Kindersberger, M Tenzer, V Hinrichsen, L Zavattoni, O Lesaint, M Muhr, and D Imamovic. Cigre 2014, d1-102. 2014.

[2] C Weijiang and L Peng. The new technological developments of uhv ac power transmission equipment. In High Voltage Engineering and Application (ICHVE), 2016 IEEE International Conference on, pages 1-6. IEEE, 2016.

[3] L Frommhold, RJ Corbin, and DW Goodson. Electron avalanches in oxygen: Theory. Physical Review A, 8(3):1403, 1973.

[4] L Frommhold. Über verzögerte Elektronen in Elektronenlawinen, insbesondere in Sauerstoff und Luft, durch Bildung und Zerfall negativer Ionen $\left(O^{-}\right)$. Fortschritte der Physik, 12(11):597-642, 1964.

[5] MA Harrison and R Geballe. Simultaneous measurement of ionization and attachment coefficients. Physical Review, 91(1):1, 1953.

[6] AN Prasad. Measurement of ionization and attachment coefficients in dry air in uniform fields and the mechanism of breakdown. Proceedings of the Physical Society, 74(1):33, 1959.

[7] SW Rayment and JL Moruzzi. Electron detachment studies between $O^{-}$ions and nitrogen. International Journal of Mass Spectrometry and Ion Physics, 26(3):321$326,1977$.

[8] HFA Verhaart and PCT Van der Laan. The influence of water vapor on avalanches in air. Journal of applied physics, 55(9):3286-3292, 1984.

[9] HFA Verhaart and PCT Van der Laan. Fast current measurements for avalanche studies. Journal of Applied Physics, 53(3):1430-1436, 1982.

[10] C Wen and JM Wetzer. Electron avalanches influenced by detachment and conversion processes. Electrical Insulation, IEEE Transactions on, 23(6):999$1008,1988$.

[11] C Wen and JM Wetzer. Time-resolved avalanche current waveforms in octafluorocyclobutane. Electrical Insulation, IEEE Transactions on, 24(1):143149, 1989.

[12] GH Friedrich. Die physikalischen Entladungsparameter der synthetischen Luft unter dem Einfluss der Feuchte und der Temperatur. Phd thesis at ETH Zurich, page 216, 1992.

[13] IA Kossyi, AY Kostinsky, AA Matveyev, and VP Silakov. Kinetic scheme of the non-equilibrium discharge in nitrogen-oxygen mixtures. Plasma Sources Science and Technology, 1(3):207, 1992.

[14] NL Aleksandrov. Three-body electron attachment to a molecule. Soviet Physics Uspekhi, 31(2):101, 1988.

[15] S Pancheshnyi. Effective ionization rate in nitrogen-oxygen mixtures. Journal of Physics D: Applied Physics, 46(15):155201, 2013.

[16] J De Urquijo, AM Juarez, JC Rodríguez-Luna, and JS Ramos-Salas. A numerical simulation code for electronic and ionic transients from a time-resolved pulsed townsend experiment. IEEE transactions on plasma science, 35(5):1204-1209, 2007. 
[17] Ge Odriozola, B Rojas, J de Urquijo, and A Bekstein. Using a parallel genetic algorithm to fit a pulsed townsend discharge simulation to experiments. In High Performance Computer Applications: 6th International Conference, ISUM 2015, Mexico City, Mexico, March 9-13, 2015, Revised Selected Papers, volume 595, page 343. Springer, 2016.

[18] DA Dahl, TH Teich, and CM Franck. Obtaining precise electron swarm parameters from a pulsed townsend setup. Journal of Physics D: Applied Physics, 45(48):485201, 2012.

[19] A Chachereau, M Rabie, and CM Franck. Electron swarm parameters of the hydrofluoroolefine HFO1234ze. Plasma Sources Science and Technology, 25(4):045005, 2016.

[20] H Zhao and H Lin. Dielectric breakdown properties of N2-O2 mixtures by considering electron detachments from negative ions. Physics of Plasmas, 23(7):073505, 2016.

[21] BC O'Neill and JD Craggs. Collisional detachment of electrons and ion molecule reactions in oxygen. Journal of Physics B: Atomic and Molecular Physics, 6(12):2625, 1973.

[22] LC Pitchford, LL Alves, K Bartschat, SF Biagi, MC Bordage, I Bray, CE Brion, MJ Brunger, L Campbell, A Chachereau, et al. Lxcat: an open-access, web-based platform for data needed for modeling low temperature plasmas. Date of retrieval: 27.04.2017. Plasma Processes and Polymers, 2016.

[23] SF Biagi. Monte carlo simulation of electron drift and diffusion in counting gases under the influence of electric and magnetic fields. Nuclear Instruments and Methods in Physics Research Section A: Accelerators, Spectrometers, Detectors and Associated Equipment, 421(1):234-240, 1999.

[24] D Smith, NG Adams, and TM Miller. A laboratory study of the reactions of $\mathrm{N}^{+}, \mathrm{N}_{2}^{+}, \mathrm{N}_{3}^{+}, \mathrm{N}_{4}^{+}, \mathrm{O}^{+}, \mathrm{O}_{2}^{+}$, and $\mathrm{NO}^{+}$ions with several molecules at $300 \mathrm{~K}$. The Journal of Chemical Physics, 69(1):308-318, 1978.

[25] RF Potter. Cross sections for charge transfer collisions of low-energy ions in $N_{2}$ and $\mathrm{O}_{2}$. The Journal of Chemical Physics, 22(6):974-979, 1954.

[26] A Bekstein, M Yousfi, M Benhenni, O Ducasse, and O Eichwald. Drift and reactions of positive tetratomic ions in dry, atmospheric air: Their effects on the dynamics of primary and secondary streamers. Journal of Applied Physics, 107(10):103308, 2010.

[27] A Bekstein, M Benhenni, M Yousfi, O Ducasse, and O Eichwald. Ion swarm data of $\mathrm{N}_{4}^{+}$in $\mathrm{N}_{2}, \mathrm{O}_{2}$ and dry air for streamer dynamics simulation. The European Physical Journal Applied Physics, 42(1):33-40, 2008.

[28] SF Biagi. Magboltz, program to compute gas transport parameters. Version, 2(2), 1997.

[29] CR Sherwood, MC Garner, KA Hanold, KM Strong, and RE Continetti. Energy and angular distributions in dissociative photodetachment of $O_{4}^{-}$. The Journal of chemical physics, 102(17):6949-6952, 1995.

[30] DC Conway and JH Yang. Bonding in homomolecular ion clusters, $O_{2 \mathrm{n}+2}^{+}$. The Journal of Chemical Physics, 43(8):2900-2902, 1965. 
[31] KA Hanold, AK Luong, and RE Continetti. Complete kinematic measurement of three-body reaction dynamics: Dissociative photodetachment of $O_{6}^{-}$. Journal of Chemical Physics, 109(21), 1998.

[32] MJ DeLuca, CC Han, and MA Johnson. Photoabsorption of negative cluster ions near the electron detachment threshold: A study of the $O_{2 n}^{-}$system. The Journal of Chemical Physics, 93(1):268-275, 1990.

[33] J de Urquijo, A Bekstein, O Ducasse, G Ruíz-Vargas, M Yousfi, and M Benhenni. Negative ion clusters in oxygen: collision cross sections and transport coefficients. The European Physical Journal D-Atomic, Molecular, Optical and Plasma Physics, 55(3):637-643, 2009.

[34] NL Aleksandrov. Three-body electron attachment to $O_{2}$ (a $\left.1 \Delta \mathrm{g}\right)$. Chemical physics letters, 212(3-4):409-412, 1993.

[35] JT Gudmundsson, IG Kouznetsov, KK Patel, and MA Lieberman. Electronegativity of low-pressure high-density oxygen discharges. Journal of Physics D: Applied Physics, 34(7):1100, 2001.

[36] RN Franklin. The role of $O_{2}(\mathrm{a} 1 \Delta \mathrm{g})$ metastables and associative detachment in discharges in oxygen. Journal of Physics D: Applied Physics, 34(12):1834, 2001.

[37] MC DeRosa and RJ Crutchley. Photosensitized singlet oxygen and its applications. Coordination Chemistry Reviews, 233:351-371, 2002.

[38] C Wen. Time-resolved swarm studies in gases with emphasis on electron detachment and ion conversion. 1989.

[39] H Böhringer, DW Fahey, W Lindinger, F Howorka, FC Fehsenfeld, and DL Albritton. Mobilities of several mass-identified positive and negative ions in air. International journal of mass spectrometry and ion processes, 81:45-65, 1987.

[40] LA Viehland and CC Kirkpatrick. Relating ion/neutral reaction rate coefficients and cross-sections by accessing a database for ion transport properties. International journal of mass spectrometry and ion processes, 149:555-571, 1995.

[41] RM Snuggs, DJ Volz, JH Schummers, DW Martin, and EW McDaniel. Mobilities and longitudinal diffusion coefficients of mass-identified potassium ions and positive and negative oxygen ions in oxygen. Physical Review A, 3(1):477, 1971.

[42] H Ryzko. Drift velocity of electrons and ions in dry and humid air and in water vapour. Proceedings of the Physical Society, 85(6):1283, 1965.

[43] W Roznerski and K Leja. Electron drift velocity in hydrogen, nitrogen, oxygen, carbon monoxide, carbon dioxide and air at moderate e/n. Journal of Physics D: Applied Physics, 17(2):279, 1984.

[44] H Hessenauer. Anlagerungskoeffizienten und Driftgeschwindigkeiten von Elektronen in Luft. Zeitschrift für Physik, 204(2):142-154, 1967.

[45] KH Wagner. Ionization, electron-attachment, -detachment, and charge-transfer in oxygen and air. Zeitschrift für Physik, 241(3):258-270, 1971.

[46] H Ryżko and E Åström. Electron attachment-detachment processes in dry air. Journal of Applied Physics, 38(1):328-330, 1967.

[47] GJM Hagelaar and LC Pitchford. Solving the boltzmann equation to obtain electron transport coefficients and rate coefficients for fluid models. Plasma Sources Science and Technology, 14(4):722, 2005. 
[48] M Rabie and CM Franck. Methes: a monte carlo collision code for the simulation of electron transport in low temperature plasmas. Computer Physics Communications, 203:268-277, 2016.

[49] C Moler and C Van Loan. Nineteen dubious ways to compute the exponential of a matrix, twenty-five years later. SIAM review, 45(1):3-49, 2003. 